Supplement of Hydrol. Earth Syst. Sci., 21, 981-997, 2017

http://www.hydrol-earth-syst-sci.net/21/981/2017/

doi:10.5194/hess-21-981-2017-supplement

(C) Author(s) 2017. CC Attribution 3.0 License.

(c) (i)

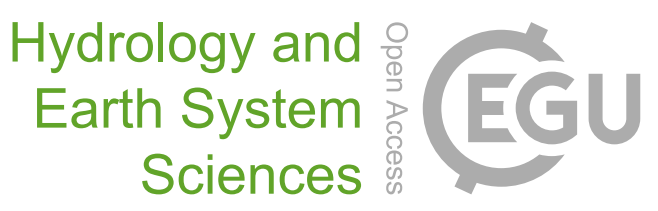

Supplement of

\title{
Water yield following forest-grass-forest transitions
}

Katherine J. Elliott et al.

Correspondence to: Katherine J. Elliott (kelliott@fs.fed.us)

The copyright of individual parts of the supplement might differ from the CC-BY 3.0 licence. 


\section{Supplement: Supporting Information}

\section{Table S1}

Sequence of treatments in Watershed 6, a north-facing 8.9 hectare catchment.

\begin{tabular}{|c|c|c|}
\hline Year & Treatment & References \\
\hline $1909-1923$ & $\begin{array}{l}\text { The Coweeta Basin, including WS6, was commercially cut } \\
\text { with ca. } 30 \% \text { reduction in basal area. Cutting progressed } \\
\text { from low to high elevation; WS6 would have been the first } \\
\text { area cut. }\end{array}$ & $\begin{array}{l}\text { Elliott and Swank, } \\
2008\end{array}$ \\
\hline 1934 & $\begin{array}{l}\text { A weir was installed at the base of the watershed, and nine } \\
\text { vegetation plots were measured across the east-side of the } \\
\text { watershed. }\end{array}$ & $\begin{array}{l}\text { Hewlett, 1961; } \\
\text { Douglass and } \\
\text { Swank, } 1972\end{array}$ \\
\hline $1928-1940$ & $\begin{array}{l}\text { Chestnut blight (Endothia parasitica Murr.) killed the } \\
\text { remaining Castanea dentata trees. }\end{array}$ & \\
\hline 1942 & $\begin{array}{l}12 \% \text { of the catchment ( } 1.1 \mathrm{ha} \text { ) along the stream was cut to } \\
\text { determine the effect of cutting streamside vegetation on } \\
\text { water yield. }\end{array}$ & $\begin{array}{l}\text { Dunford and } \\
\text { Fletcher, } 1947\end{array}$ \\
\hline 1958 , summer & $\begin{array}{l}\text { Vegetation was measured in } 37 \text { plots prior to grass } \\
\text { conversion. }\end{array}$ & \\
\hline 1958, fall & $\begin{array}{l}\text { All merchantable timber was cut and removed. All } \\
\text { remaining vegetation was cut, piled and burned. A seedbed } \\
\text { was prepared by burning and soil scarification. }\end{array}$ & Hibbert ,1969 \\
\hline 1959 & $\begin{array}{l}\text { Grass conversion by application of } 1100 \mathrm{~kg} \mathrm{ha}^{-1} \text { limestone, } \\
366 \mathrm{~kg} \mathrm{ha}^{-1} \mathrm{NPK} \text { fertilizer, and } 3.7 \mathrm{~kg} \mathrm{ha}^{-1} \text { fescue (Festuca } \\
\text { octiflora Walter) grass seed across the entire watershed. }\end{array}$ & Hibbert, 1969 \\
\hline 1960-1965 & $\begin{array}{l}\text { Woody sprouts were foliar sprayed with 2, 4, 5-T herbicide, } \\
\text { annually for five years. }\end{array}$ & \\
\hline 1960 & Application of $37 \mathrm{~kg} \mathrm{ha}^{-1} \mathrm{NH}_{4}-\mathrm{NO}_{3}$ & \\
\hline 1965 & $\begin{array}{l}\text { Application of } 110 \mathrm{~kg} \mathrm{ha}^{-1} \mathrm{NPK} \text { fertilizer and } 27 \mathrm{~kg} \mathrm{ha}^{-1} \\
60 \% \text { potash. }\end{array}$ & \\
\hline 1966- early & Three herbicides were used to eliminate the grass cover. & Hibbert, 1969 \\
\hline 1967 & $\begin{array}{l}\text { Atrazine, paraquat, and 2,4-D were applied on the entire } \\
\text { watershed except for a } 3 \mathrm{~m} \text { buffer strip on each side of the } \\
\text { perennial stream. }\end{array}$ & \\
\hline 1967 & $\begin{array}{l}\text { No further treatments, vegetation was allowed to revert to } \\
\text { successional vegetation. For first several growing seasons, } \\
\text { the entire area was dominated by a dense cover of } \\
\text { herbaceous species. }\end{array}$ & $\begin{array}{l}\text { Burt and Swank, } \\
1992\end{array}$ \\
\hline 1968 & $\begin{array}{l}\text { Vegetation was measured in } 34 \text { plots, the first growing } \\
\text { season following treatment abandonment. }\end{array}$ & $\begin{array}{l}\text { Burt and Swank, } \\
1992\end{array}$ \\
\hline 1982 & 15-year-old forest & Elliott et al., 1998 \\
\hline 1995 & 28-year-old forest & Elliott et al., 1998 \\
\hline 2012 & 45-year-old forest & This study. \\
\hline
\end{tabular}




\section{Table S2}

Common tree species across the eastern United States classified by hydrotype (mesophytic, semi-mesophytic, xerophytic) ${ }^{\mathrm{a}}$, xylem anatomy (diffuse-porous, ring-porous, semi-ring-porous, or tracheid) $)^{\mathrm{b}}$, and stomatal control (isohydric or anisohydric) ${ }^{\mathrm{c}}$.

\begin{tabular}{|c|c|c|c|}
\hline Species & Hydrotype $^{\mathrm{a}}$ & $\begin{array}{l}\text { Xylem } \\
\text { anatomy }\end{array}$ & $\begin{array}{l}\text { Stomatal } \\
\text { control }^{\mathrm{c}} \\
\end{array}$ \\
\hline Acer rubrum & Mesophytic & Diffuse & Isohydric \\
\hline Acer saccharum & Mesophytic & Diffuse & Isohydric \\
\hline Acer pensylvanicum & Mesophytic & Diffuse & Isohydric \\
\hline Betula alleghaniensis & Mesophytic & Diffuse & Isohydric \\
\hline Betula lenta & Mesophytic & Diffuse & Isohydric \\
\hline Betula papyrifera & Mesophytic & Diffuse & Isohydric \\
\hline Carya spp. & Semi-mesophytic & Semi-ring & Intermediate \\
\hline Castanea dentata & Semi-meophytic & Semi-ring & Anisohydric \\
\hline Fagus grandifolia & Mesophytic & Diffuse & Isohydric \\
\hline $\begin{array}{l}\text { Fraxinus spp. (americana, } \\
\text { pennsylvanica) }\end{array}$ & Mesophytic & Ring & Anisohydric \\
\hline Liriodendron tulipifera & Mesophytic & Diffuse & Isohydric \\
\hline $\begin{array}{l}\text { Magnolia spp. (acuminata, } \\
\text { fraseri) }\end{array}$ & Mesophytic & Diffuse & Isohydric \\
\hline Nyssa sylvatica & Semi-mesophytic & Diffuse & Intermediate \\
\hline Oxydendron arborea & Semi-mesophytic & Ring & Intermediate \\
\hline $\begin{array}{l}\text { Pinus spp. (rigida, taeda, } \\
\text { echinata, pungens, virginiana) }\end{array}$ & Xerophytic & Tracheid & Isohydric \\
\hline Pinus palustris & Xerophytic & Tracheid & Isohydric \\
\hline Pinus strobus & Semi-mesophytic & Tracheid & Isohydric \\
\hline Prunus serotina & Semi-mesophytic & Ring & Anisohydric \\
\hline Quercus alba & Xerophytic & Ring & Anisohydric \\
\hline Quercus coccinea & Xerophytic & Ring & Anisohydric \\
\hline Quercus montana & Xerophytic & Ring & Anisohydric \\
\hline Quercus rubra & Semi-mesophytic & Ring & Anisohydric \\
\hline Quercus velutina & Semi-mesophytic & Ring & Anisohydric \\
\hline Robinia pseudoacacia & Semi- mesophytic & Ring & Isohydric \\
\hline Tilia americana & Mesophytic & Diffuse & Isohydric \\
\hline Tsuga canadensis & Mesophytic & Tracheid & Anisohydric \\
\hline
\end{tabular}

Classifications were extracted from the literature; ahydrotype (Flatley et al., 2015), bylem anatomy (Ewers et al., 2007) and 'stomatal control (Roman et al., 2015); ' stomatal control of Castanea dentata based on C. sativa (GomesLaranjo et al., 2012). 


\section{Table S3}

Mean density, basal area, total aboveground biomass, and leaf area index (LAI) for the treated (WS6) and reference (WS14, WS18) watersheds overtime. Standard errors are in parentheses.

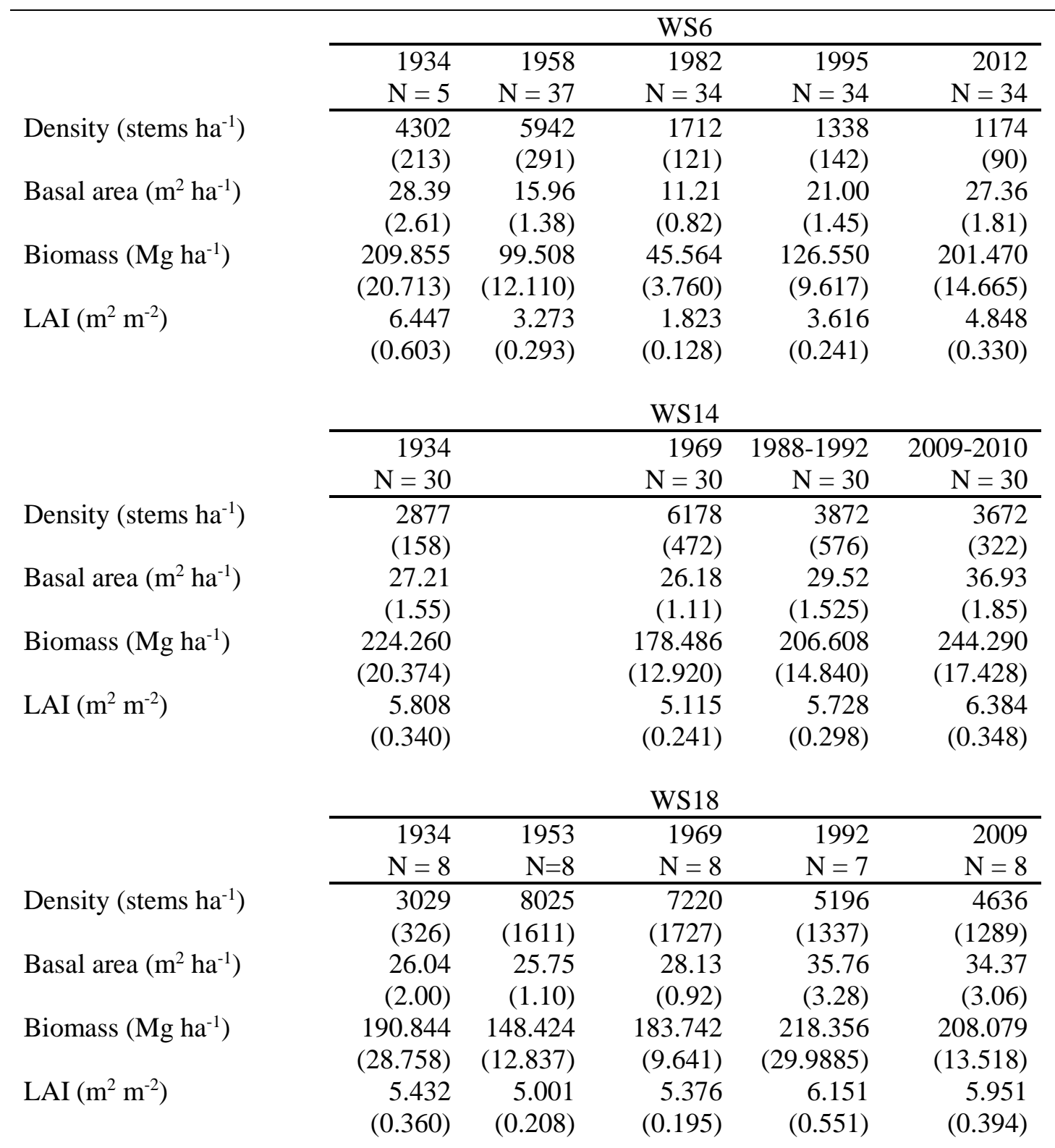

In 1934, only percent cover of evergreen shrubs (Kalmia latifolia and Rhododendron maximum) was estimated for each plot. For all other years, diameter of evergreen shrubs was measured. The higher density in years 1953 and 1958 versus 1934 is partly due to evergreen shrub diameters not being measured or counted in the permanent plots in 1934. 
Table S4

Watershed 14: mean density, basal area, aboveground biomass, and leaf area index (LAI) of species over time [1934, 1969,

1988-1992, and 2009-2010]. Standard errors are in parentheses.

\begin{tabular}{|c|c|c|c|c|c|}
\hline \multirow[b]{2}{*}{ Species in 1934} & \multirow{2}{*}{$\begin{array}{c}\text { Density } \\
\left(\text { stems ha }^{-1}\right)\end{array}$} & \multirow{2}{*}{$\begin{array}{l}\text { Basal area } \\
\left(\mathrm{m}^{2} \mathrm{ha}^{-1}\right)\end{array}$} & \multicolumn{2}{|c|}{ Aboveground biomass ( $\mathrm{kg} \mathrm{ha}^{-1}$ ) } & \multirow{2}{*}{$\begin{array}{c}\text { LAI } \\
\left(\mathrm{m}^{2} \mathrm{~m}^{-2}\right)\end{array}$} \\
\hline & & & Leaf & Total & \\
\hline Castanea dentata & 764 (119) & $12.50(1.44)$ & 1,509 (175) & 120,549 (17775) & $2.506(0.291)$ \\
\hline Quercus montana & 269 (43) & 3.79 (0.79) & 576 (129) & 43,036 (11489) & $0.818(0.184)$ \\
\hline Carya spp. & $228(30)$ & $1.62(0.30)$ & $196(44)$ & 11,991 (3710) & $0.369(0.084)$ \\
\hline Quercus coccinea & 164 (37) & $1.74(0.36)$ & 488 (97) & $11,225(2596)$ & $0.605(0.122)$ \\
\hline Acer rubrum & 234 (24) & $1.22(0.17)$ & 222 (28) & 6,664 (1171) & $0.381(0.048)$ \\
\hline Nyssa sylvatica & $108(14)$ & $0.96(0.22)$ & 68 (11) & $5,791(1814)$ & $0.166(0.028)$ \\
\hline Quercus velutina & $56(12)$ & $0.79(0.19)$ & 101 (27) & $5,112(1501)$ & $0.126(0.034)$ \\
\hline Quercus alba & $29(15)$ & $0.45(0.22)$ & 59 (25) & $3,347(2162)$ & $0.087(0.037)$ \\
\hline Robinia pseudoacacia & 71 (9) & $0.58(0.09)$ & 68 (11) & $2,888 \quad(604)$ & $0.090(0.015)$ \\
\hline Quercus rubra & 40 (9) & $0.46(0.13)$ & 58 (19) & $2,850(1146)$ & $0.084(0.026)$ \\
\hline Tsuga canadensis & $32(15)$ & $0.48(0.26)$ & 100 (48) & $2,512(1384)$ & $0.077(0.038)$ \\
\hline Betula lenta & $128(56)$ & $0.41(0.15)$ & 21 & $1,865(820)$ & $0.055(0.024)$ \\
\hline Liriodendron tulipifera & $181(70)$ & $0.48(0.16)$ & 41 (13) & 1,429 (444) & $0.075(0.024)$ \\
\hline Oxydendrum arboreum & $50(8)$ & $0.32(0.07)$ & 29 & 1,139 (270) & $0.073(0.014)$ \\
\hline Cornus florida & $121(24)$ & $0.32(0.07)$ & 35 & 761 (186) & $0.076(0.015)$ \\
\hline Sassafras albidum & $112(16)$ & $0.27(0.04)$ & $32 \quad(5)$ & 723 (165) & $0.051(0.009)$ \\
\hline Hamamelis virginiana & $113(35)$ & $0.23(0.07)$ & $27 \quad(8)$ & 517 (159) & $0.056(0.017)$ \\
\hline Castanea pumila & $63(16)$ & $0.14(0.04)$ & $17 \quad$ (4) & $353 \quad(101)$ & $0.027(0.007)$ \\
\hline Tilia americana & $2(1)$ & $0.04(0.04)$ & (4) & $306 \quad(300)$ & $0.009(0.008)$ \\
\hline Pinus rigida & $2(2)$ & $0.08(0.08)$ & $4 \quad(4)$ & $300 \quad(300)$ & $0.009(0.009)$ \\
\hline Amelanchier arborea & $31(10)$ & $0.08(0.03)$ & $9 \quad(3)$ & $213 \quad(81)$ & $0.015(0.005)$ \\
\hline Acer pensylvanicum & $28(11)$ & $0.08(0.03)$ & $14 \quad(6)$ & 204 (88) & $0.029(0.010)$ \\
\hline Magnolia acuminata & $2(1)$ & $0.03(0.03)$ & $4 \quad(3)$ & 180 (174) & $0.011(0.010)$ \\
\hline Magnolia fraseri & $6(4)$ & $0.02(0.02)$ & (4) & 104 (84) & $0.023(0.014)$ \\
\hline Fagus grandifolia & $3(2)$ & $0.01(0.01)$ & (1) & $62 \quad(56)$ & $0.003(0.002)$ \\
\hline Symplocos tinctoria & $12(7)$ & $0.02(0.01)$ & (2) & $51 \quad$ (31) & $0.004(0.003)$ \\
\hline Aesculus flava & 9 (5) & $0.02(0.01)$ & 2 (1) & (24) & $0.004(0.002)$ \\
\hline Fraxinus americana & 5 (3) & $0.01(0.01)$ & $1(0.7)$ & $24 \quad(12)$ & $0.005(0.003)$ \\
\hline
\end{tabular}


Quercus falcata

Diospyros virginiana

Salix nigra

Linder benzoin

All Quercus

Total

\section{Species in 1969}

Quercus montana

Quercus coccinea

Acer rubrum

Carya spp.

Quercus rubra

Liriodendron tulipifera

Betula lenta

Quercus velutina

Tsuga canadensis

Kalmia latifolia

Quercus alba

Rhododendron maximum

Robinia pseudoacacia

Nyssa sylvatica

Oxydendrum arboreum

Cornus florida

Magnolia acuminata

Castanea dentata

Amelanchier arborea

Fagus grandifolia

Tilia americana

Pinus rigida

Acer pensylvanicum

Sassafras albidum

Hamamelis virginiana

Aesculus flava

Magnolia fraseri

$\begin{array}{rr}0.8(0.6) & 0.002(0.001) \\ 0.8(0.8) & 0.002(0.002) \\ 5(4) & 0.01(0.01) \\ 2 \quad(2) & 0.005(0.004) \\ 560(65) & 7.24(0.93) \\ 2877(158) & 27.21(1.55)\end{array}$

2 (1)

$0.2(0.2)$

$0.03(0.02)$

$0.01(0.01)$

1,284 (168)

3,699 (213)

836 (175)

418 (85)

388 (49)

201 (58)

$230(51)$

116 (34)

69 (24)

$112(41)$

226 (97)

239 (51)

78 (32)

$220(43)$

93 (20)

44 (9)

60 (11)

65 (16)

19 (8)

33 (9)

26 (7)

14 (11)

10 (9)

10 (10)

22 (8)

15 (4)

12 (6)

5 (3)

5 (2)
$65,576(11941)$
$224,260(20374)$

$\begin{array}{rr}5 & (3) \\ 4 & (4) \\ 0.03 & (0.02) \\ 0.01 & (0.01) \\ & \\ & \\ & \\ & \end{array}$

0.002 (0.001)

$0.0003(0.0003)$

$0.002(0.001)$

$<0.0001$

$1.736(0.231)$

$5.808(0.340)$

$\begin{array}{rr}56,570(13468) \\ 19,432(4697) \\ 15,618(2539) \\ 13,069(4446) \\ 12,420(3080) \\ 7,270(2190) \\ 6,332(2235) \\ 6,284(2465) \\ 5,688(2537) \\ 5,268(1124) \\ 5,020(2667) \\ 5,539(1102) \\ 4,640(1074) \\ 3,780(1353) \\ 3,619 & (668) \\ 1,718 & (470) \\ 1,142 & (517) \\ 1,069 & (552) \\ 747 & (228) \\ 656 & (530) \\ 619 & (606) \\ 597 & (597) \\ 485 & (188) \\ 472 & (119) \\ 256 & (119) \\ 215 & (125) \\ 155 & (64)\end{array}$

$1.188(0.248)$

$0.535(0.108)$

$0.678(0.086)$

$0.330(0.095)$

$0.295(0.066)$

$0.214(0.062)$

$0.188(0.066)$

$0.143(0.052)$

$0.180(0.078)$

$0.184(0.038)$

$0.115(0.046)$

$0.170(0.033)$

$0.126(0.027)$

$0.111(0.024)$

$0.158(0.028)$

$0.131(0.033)$

$0.064(0.027)$

$0.055(0.015)$

$0.044(0.012)$

$0.024(0.018)$

$0.016(0.015)$

$0.019(0.019)$

$0.039(0.013)$

$0.024(0.007)$

$0.025(0.012)$

$0.009(0.005)$

0.017 (0.006) 
Castanea pumila

Fraxinus americana

Diospyros virginiana

Symplocos tinctoria

$R$. calendulaceum

Pyrularia pubera

Calycanthus florida

Ilex opaca

All Quercus

Total

Species in 1988-1992

Quercus montana

Acer rubrum

Quercus rubra

Quercus coccinea

Tsuga canadensis

Liriodendron tulipifera

Betula lenta

Carya spp.

Quercus velutina

Oxydendrum arboreum

Robinia pseudoacacia

Nyssa sylvatica

Rhododendron maximum

Kalmia latifolia

Cornus florida

Magnolia acuminata

Tilia americana

Amelanchier arborea

Quercus alba

Acer saccharum

Sassafras albidum
$372(43)$

$6178(472)$

$16 \quad(7)$

2 (1)

8 (7)

5 (2)

79 (21)

28 (11)

5 (3)

2 (2)

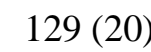

147 (19)

25 (5)

46 (11)

45 (16)

62 (15)

70 (19)

62 (16)

11 (4)

$43 \quad$ (7)

21 (3)

44 (8)

1300 (350)

1476 (434)

113 (34)

5 (2)

4 (3)

32 (8)

9 (5)

10 (6)

15 (4)
$0.04(0.02)$

$0.02(0.02)$

$0.02(0.02)$

$0.01(0.004)$

$0.16(0.04)$

$0.06(0.02)$

$0.01(0.01)$

$0.01(0.01)$

11.25 (1.26)

26.18 (1.11)

$5.40(0.98)$

$3.70(0.54)$

$2.14(0.52)$

$2.39(0.61)$

$2.53(1.10)$

$2.11(0.56)$

$1.60(0.51)$

$0.89(0.24)$

$0.61(0.31)$

$1.09(0.19)$

$0.68(0.16)$

$0.78(0.20)$

$1.51(0.31)$

$0.89(0.25)$

$0.76(0.23)$

$0.27(0.16)$

$0.29(0.18)$

$0.29(0.08)$

$0.19(0.12)$

$0.17(0.10)$

$0.20(0.07)$
5 (2)

3 (2)

3 (3)

$1(0.4)$

$0.4(0.1)$

$0.15(0.06)$

$0.02(0.02)$

$0.02(0.02)$

1,674 (197)

3,587 (168)

808 (156)

574 (79)

321 (83)

342 (80)

423 (175)

175 (47)

120 (39)

115 (34)

91 (49)

76 (13)

81 (19)

$54 \quad(12)$

152 (37)

$150 \quad(43)$

88 (27)

32 (21)

$35 \quad(22)$

35 (10)

$26 \quad(16)$

38 (17)

$23 \quad(9)$

$$
\begin{array}{rr}
112 & (60) \\
102 & (73) \\
84 & (80) \\
22 & (8) \\
0.4 & (0.1) \\
0.15 & (0.06) \\
0.03 & (0.02) \\
0.02 & (0.02)
\end{array}
$$

99,728 (13973)

178,486 (12920)

53,796 (12688)

28,991 (4924)

18,536 (5138)

18,224 (4963)

$13,731(6000)$

13,006 (3573)

11,006 (3595)

7,273 (2424)

5,289 (2920)

5,202 (924)

4,333 (1164)

4,127 (1282)

3,443 (832)

3,313 (965)

3,300 (1291)

2,170 (1390)

2,088 (1298)

$1,402 \quad(472)$

1,133 (760)

$1,050 \quad$ (665)

964 (422)
$5.114(0.241)$

$0.008(0.004)$

$0.005(0.003)$

$0.005(0.004)$

$0.002(0.001)$

$0.001(0.0002)$

$0.0002(0.0001)$

$<0.0001$

$<0.0001$

$2.275(0.273)$

$1.147(0.221)$

$1.004(0.138)$

$0.411(0.106)$

$0.438(0.103)$

$0.357(0.150)$

$0.324(0.086)$

$0.325(0.105)$

$0.188(0.055)$

$0.117(0.062)$

$0.197(0.034)$

$0.111(0.026)$

0.137 (0.029)

$0.117(0.029)$

$0.116(0.034)$

$0.177(0.055)$

$0.107(0.066)$

$0.058(0.036)$

0.058 (0.016)

$0.039(0.024)$

$0.049(0.030)$

$0.039(0.015)$ 
Pinus rigida

Castanea dentata

Hamamelis virginiana

Aesculus flava

Magnolia fraseri

Acer pensylvanicum

Diospyros virginiana

Fraxinus americana

Castanea pumila

Fagus grandifolia

$R$. calendulaceum

Pyrularia pubera

All Quercus

Total

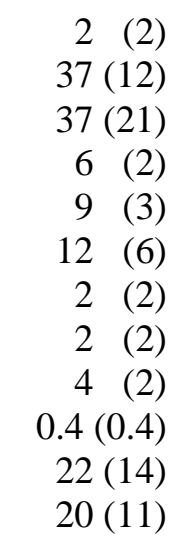

$220 \quad(28)$

3872 (576)

Species in 2009-2010

Quercus montana

Acer rubrum

Quercus coccinea

Quercus rubra

Liriodendron tulipifera

Betula lenta

Carya spp.

Kalmia latifolia

Oxydendrum arboretum

Rhododendron maximum

Nyssa sylvatica

Quercus alba

Magnolia acuminata

Amelanchier arborea

Quercus velutina

Magnolia fraseri

Robinia pseudoacacia

Tilia americana

Sassafras albidum

111 (19)

144 (18)

42 (8)

22 (5)

49 (12)

43 (11)

55 (14)

1728 (342)

38 (6)

1159 (206)

39 (8)

8 (4)

4 (2)

40 (10)

6 (3)

19 (4)

11 (2)

1 (1)

10 (2)

$\begin{array}{rrr}0.16(0.15) & 16 & (14) \\ 0.15(0.07) & 17 & (9) \\ 0.13(0.06) & 15 & (7) \\ 0.08(0.03) & 9 & (4) \\ 0.08(0.03) & 19 & (10) \\ 0.07(0.03) & 14 & (6) \\ 0.04(0.03) & 5 & (4) \\ 0.01(0.01) & 1(0.8) \\ 0.01(0.005) & 0.94(0.53) \\ 0.001(0.001) & 0.10(0.10) \\ 0.05(0.03) & 0.12(0.08) \\ 0.04(0.02) & 0.11(0.06)\end{array}$

10.72 (1.34)

29.52 (1.52)

$1,589(204)$
$3,861(195)$

6.96 (1.17)
$4.61(0.60)$

$3.12(0.62)$

$2.08(0.50)$

$2.48(0.65)$

$1.71(0.51)$

$1.19(0.33)$

$4.35(0.83)$

$1.42(0.23)$

$4.47(0.82)$

$0.90(0.21)$

$0.50(0.28)$

$0.36(0.20)$

$0.54(0.14)$

$0.38(0.21)$

$0.35(0.11)$

$0.36(0.10)$

$0.16(0.12)$

$0.15(0.05)$

$$
\begin{array}{rr}
830 & (808) \\
824 & (638) \\
404 & (184) \\
378 & (180) \\
350 & (136) \\
342 & (169) \\
295 & (267) \\
30 & (25) \\
18 & (10) \\
2 & (2) \\
0.13 & (0.08) \\
0.12 & (0.07)
\end{array}
$$

96,981 (15036)

206,608 (14840)

$$
\begin{array}{r}
1060(185) \\
709(89) \\
387(78) \\
311(79) \\
204(53) \\
155(47) \\
156(47) \\
319(62) \\
93(15) \\
257(46) \\
60(13) \\
58(29) \\
42(24) \\
64(17) \\
48(26) \\
41(13) \\
42(12) \\
18(14) \\
18(6)
\end{array}
$$

$0.027(0.027)$

$0.029(0.014)$

$0.030(0.014)$

$0.015(0.007)$

$0.032(0.011)$

$0.024(0.010)$

$0.009(0.007)$

$0.002(0.001)$

$0.002(0.001)$

0.0002 (0.0002)

$0.0002(0.0001)$

0.0002 (0.0001)

$2.153(0.281)$

$5.728(0.298)$

$1.505(0.263)$

$1.240(0.156)$

$0.496(0.100)$

$0.399(0.102)$

$0.379(0.098)$

$0.422(0.128)$

$0.255(0.077)$

$0.250(0.048)$

$0.240(0.038)$

$0.201(0.035)$

$0.152(0.034)$

$0.086(0.043)$

$0.143(0.081)$

0.106 (0.029)

$0.064(0.036)$

$0.139(0.045)$

$0.058(0.017)$

0.031 (0.024)

0.029 (0.009) 


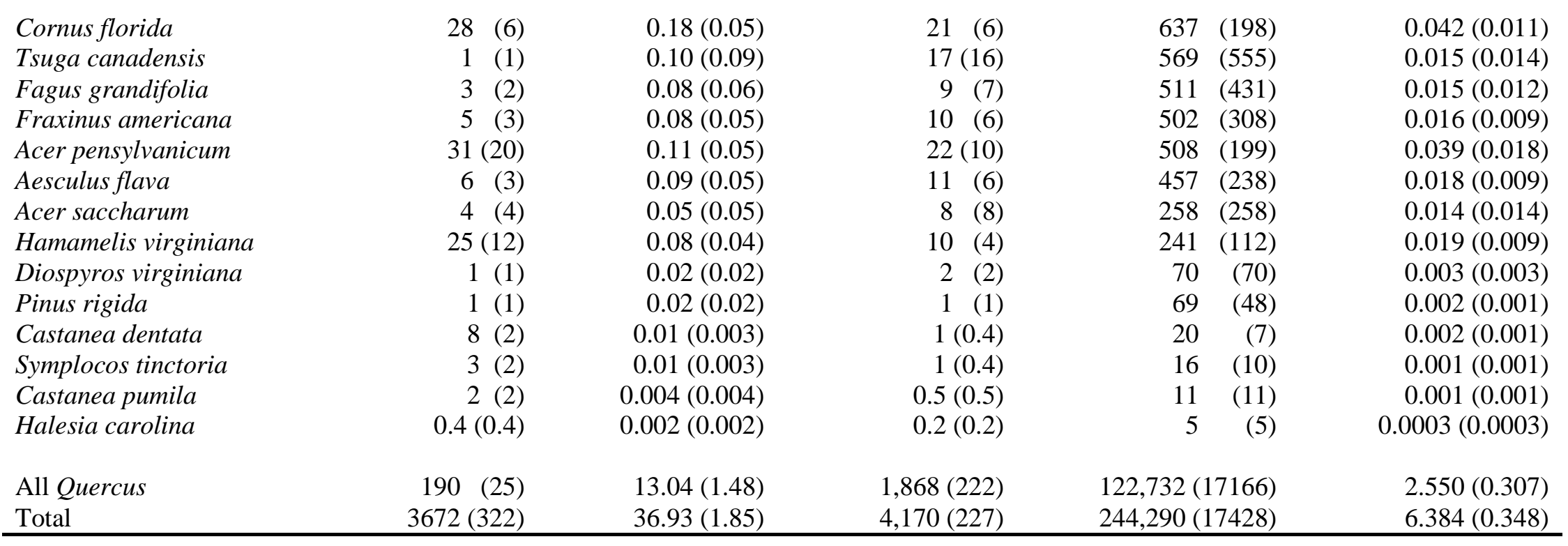

In 1934, only percent cover of evergreen shrubs (Kalmia latifolia and Rhododendron maximum) was estimated for each plot. For all other years, diameter of evergreen shrubs was measured. 


\section{Table S5}

Watershed 18: mean density, basal area, aboveground biomass, and leaf area index (LAI) of species over time [1934, 1953, 1969,

1992, and 2009]. Standard errors are in parentheses.

\begin{tabular}{|c|c|c|c|c|c|}
\hline & Density & Basal area & \multicolumn{2}{|c|}{ Aboveground biomass $\left(\mathrm{kg} \mathrm{ha}^{-1}\right)$} & \multirow{2}{*}{$\begin{array}{c}\mathrm{LAI} \\
\left(\mathrm{m}^{2} \mathrm{~m}^{-2}\right)\end{array}$} \\
\hline Species in 1934 & $\left(\right.$ stems ha $\left.{ }^{-1}\right)$ & $\left(\mathrm{m}^{2} \mathrm{ha}^{-1}\right)$ & Leaf & Total & \\
\hline Castanea dentata & $581(149)$ & $7.99(1.51)$ & $963(186)$ & $76,843(23586)$ & $1.599(0.309)$ \\
\hline Quercus montana & $273(48)$ & 3.54 (1.19) & 525 (193) & 34,656 (15047) & $0.745(0.275)$ \\
\hline Quercus rubra & 45 (17) & $2.45(1.13)$ & 359 (169) & 20,131 (9619) & $0.460(0.216)$ \\
\hline Quercus coccinea & $226(36)$ & $2.71(0.70)$ & $716(152)$ & $16,980(4885)$ & $0.917(0.195)$ \\
\hline Nyssa sylvatica & $167(30)$ & $1.76(0.76)$ & 112 (30) & $12,220(7618)$ & $0.285(0.077)$ \\
\hline Pinus rigida & 66 (47) & $1.37(1.02)$ & 94 (74) & 5,785 (4459) & $0.189(0.147)$ \\
\hline Carya spp. & 344 (119) & $1.27(0.39)$ & $120(40)$ & $5,551(2191)$ & $0.197(0.066)$ \\
\hline Quercus velutina & $197(742)$ & $1.30(0.53)$ & 132 (55) & $5,275(2211)$ & $0.169(0.070)$ \\
\hline Robinia pseudoacacia & 95 (16) & $0.76(0.27)$ & $91 \quad(32)$ & $5,031(2281)$ & $0.124(0.044)$ \\
\hline Acer rubrum & 270 (64) & $0.65(0.16)$ & 140 (33) & $2,237 \quad(744)$ & $0.245(0.059)$ \\
\hline Cornus florida & 305 (145) & $0.83(0.39)$ & 93 (44) & $2,135(1001)$ & $0.187(0.088)$ \\
\hline Oxydendrum arboreum & 109 (24) & $0.57(0.13)$ & 56 (12) & $1,789(450)$ & $0.146(0.032)$ \\
\hline Castanea pumila & $211(104)$ & $0.43(0.21)$ & $50 \quad(24)$ & 928 (465) & $0.081(0.040)$ \\
\hline Quercus alba & $9 \quad(6)$ & $0.16(0.15)$ & 24 (22) & 738 (723) & $0.036(0.033)$ \\
\hline Sassafras albidum & 62 (15) & $0.13(0.04)$ & $14 \quad(4)$ & $(67)$ & $0.051(0.009)$ \\
\hline Hamamelis virginiana & 56 (43) & $0.11(0.10)$ & 13 (12) & 248 (232) & $0.056(0.017)$ \\
\hline Liriodendron tulipifera & $28 \quad(24)$ & $0.06(0.04)$ & $(4)$ & 120 (105) & $0.075(0.024)$ \\
\hline Amelanchier arborea & $2 \quad(2)$ & $0.003(0.003)$ & $0.04(0.04)$ & (7) & $0.001(0.001)$ \\
\hline Diospyros tinctoria & (2) & $0.003(0.003)$ & $0.04(0.04)$ & (7) & $0.001(0.001)$ \\
\hline Linder benzoin & $6 \quad(6)$ & $0.01(0.01)$ & $0.03(0.03)$ & $<1$ & $<0.001$ \\
\hline All Quercus & 752 (105) & $10.16(1.22)$ & 1,757 (199) & 77,782 (15104) & 2.327 (0.279) \\
\hline Total & $3030(326)$ & $26.04(2.00)$ & 3,503 (221) & 190,845 (28758) & $5.432(0.360)$ \\
\hline \multicolumn{6}{|l|}{ Species in 1953} \\
\hline Quercus montana & $293(92)$ & $3.73(1.33)$ & $546(214)$ & 35,634 (17030) & $0.776(0.304)$ \\
\hline Quercus rubra & 31 (10) & $2.62(1.26)$ & 395 (197) & 22,667 (11671) & $0.506(0.252)$ \\
\hline Quercus coccinea & $153(36)$ & 3.34 (0.79) & $664(136)$ & 22,541 (6158) & $0.851(0.174)$ \\
\hline
\end{tabular}


Kalmia latifolia

Carya spp.

Pinus rigida

Quercus velutina

Nyssa sylvatica

Acer rubrum

Robinia pseudoacacia

Cornus florida

Oxydendrum arboreum

Liriodendron tulipifera

Quercus marilandica

Quercus alba

Castanea pumila

Symplocos tinctoria

Castanea dentata

Sassafras albidum

Hamamelis virginiana

Rhododendron maximum

Juglans cinerea

Tsuga canadensis

Acer saccharum

Amelanchier arborea

Diospyros virginiana

Rhodo. calendulaceum

\section{All Quercus}

Total

$651(119)$
$8025(1611)$

\section{Species in 1969}

Quercus montana

Quercus rubra

Quercus coccinea

Kalmia latifolia

Pinus rigida

Quercus velutina

Carya spp.

$\begin{array}{rr}5173(1833) \\ 362(124) \\ 66 & (42) \\ 145 & (742) \\ 172 & (24) \\ 301 & (71) \\ 45 & (6) \\ 326 & (148) \\ 106 & (19) \\ 81 & (60) \\ 12 & (12) \\ 16 & (11) \\ 136 & (52) \\ 101 & (34) \\ 102 & (14) \\ 72 & (32) \\ 73 & (72) \\ 73 & (27) \\ 12 & (7) \\ 2 & (2) \\ 2 & (2) \\ 2 & (2) \\ 2 & (2) \\ 162 & (105) \\ & \\ 651 & (119) \\ 8025 & (1611)\end{array}$

$$
\begin{array}{r}
2.87(0.91) \\
1.90(0.65) \\
2.16(1.38) \\
1.45(0.53) \\
1.15(0.30) \\
1.05(0.31) \\
0.70(0.21) \\
1.20(0.56) \\
0.95(0.16) \\
0.56(0.41) \\
0.31(0.31) \\
0.25(0.24) \\
0.28(0.11) \\
0.21(0.07) \\
0.21(0.03) \\
0.16(0.06) \\
0.15(0.14) \\
0.10(0.06) \\
0.02(0.01) \\
0.003(0.003) \\
0.003(0.003) \\
0.003(0.003) \\
0.003(0.003) \\
0.33(0.21) \\
11.71(0.84) \\
25.75(1.10)
\end{array}
$$

\begin{tabular}{|c|c|}
\hline 11,464 (3935) & 0.402 (0.139) \\
\hline $10,643(4624)$ & $0.331(0.125)$ \\
\hline 9,690 (6329) & $0.319(0.210)$ \\
\hline 6,653 (3079) & $0.202(0.089)$ \\
\hline 5,247 (1738) & $0.239(0.048)$ \\
\hline 4,482 (1940) & $0.365(0.096)$ \\
\hline 4,425 (1765) & $0.114(0.034)$ \\
\hline 3,661 (1702) & $0.274(0.128)$ \\
\hline 3,483 (617) & $0.216(0.038)$ \\
\hline 2,240 (1550) & $0.087(0.064)$ \\
\hline 1,695 (1695) & $0.047(0.047)$ \\
\hline 1,354 (1333) & $0.055(0.051)$ \\
\hline 635 (261) & $0.054(0.022)$ \\
\hline 483 (165) & $0.041(0.014)$ \\
\hline $448 \quad(61)$ & 0.039 (0.005) \\
\hline 353 (140) & $0.030(0.012)$ \\
\hline $324 \quad(316)$ & $0.035(0.033)$ \\
\hline $207 \quad(92)$ & $0.007(0.001)$ \\
\hline $53 \quad(30)$ & $0.003(0.001)$ \\
\hline $12 \quad(12)$ & $0.001(0.001)$ \\
\hline (9) & $0.001(0.001)$ \\
\hline (7) & $0.001(0.001)$ \\
\hline $7 \quad$ (7) & $0.001(0.001)$ \\
\hline $0.9(0.6)$ & $0.001(0.001)$ \\
\hline נתכנו & $2.437(0.233)$ \\
\hline 48,424 (12837) & $5.001(0.208)$ \\
\hline
\end{tabular}

1,838 (165) 3,507 (207)

605 (219)

584 (307)

664 (161)

518 (180)

185 (125)

221 (121)

171 (91)
90,547 (11999) 148,424 (12837)

40,050 (17662)

$0.860(0.310)$ 0.747 (0.393)

$0.850(0.206)$

$0.399(0.139)$

$0.356(0.239)$

$0.282(0.155)$

0.281 (0.149) 
Nyssa sylvatica

Acer rubrum

Oxydendrum arboreum

Liriodendron tulipifera

Robinia pseudoacacia

Cornus florida

Quercus alba

Rhododendron maximum

Castanea dentata

Acer pensylvanicum

Sassafras albidum

Symplocos tinctoria

Hamamelis virginiana

Castanea pumila

Tsuga canadensis

Amelanchier arborea

Magnolia acuminata

Magnolia fraseri

R. calendulaceum

Pyrularia pubera

All Quercus

Total

\section{Species in 1992}

Quercus montana

Quercus coccinea

Quercus rubra

Acer rubrum

Kalmia latifolia

Carya spp.

Pinus rigida

Liriodendron tulipifera

Quercus velutina

Oxydendrum arboreum

Nyssa sylvatica

$\begin{array}{rr}72 & (12) \\ 265 & (63) \\ 131 & (31) \\ 52 & (36) \\ 34 & (12) \\ 248 & (102) \\ 12 & (9) \\ 194 & (84) \\ 72 & (36) \\ 14 & (12) \\ 52 & (23) \\ 50 & (23) \\ 56 & (51) \\ 30 & (15) \\ 2 & (2) \\ 2 & (2) \\ 2 & (2) \\ 2 & (2) \\ 71 & (55) \\ 3 & (3)\end{array}$

448 (83)

7220 (1727)

$$
\begin{array}{r}
1.07(0.26) \\
1.21(0.31) \\
1.44(0.27) \\
0.99(0.72) \\
0.62(0.22) \\
1.03(0.48) \\
0.30(0.29) \\
0.20(0.10) \\
0.14(0.07) \\
0.07(0.06) \\
0.12(0.05) \\
0.11(0.05) \\
0.11(0.10) \\
0.06(0.03) \\
0.003(0.003) \\
0.003(0.003) \\
0.003(0.003) \\
0.003(0.003) \\
0.16(0.04) \\
0.05(0.02) \\
\\
14.37(1.10) \\
28.13(0.92)
\end{array}
$$

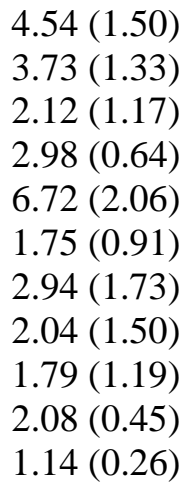

4.54 (1.50)

52 (13)

16 (6)

304 (52)

3859 (1309)

71 (26)

48 (30)

36 (28)

30 (14)

93 (24)

64 (17)

$$
\begin{array}{r}
72(14) \\
229(54) \\
118(22) \\
83(60) \\
74(25) \\
116(54) \\
42(40) \\
22(10) \\
17(8) \\
14(13) \\
14(6) \\
12(6) \\
13(12) \\
7(3) \\
2(2) \\
0.4(0.4) \\
0.4(0.4) \\
0.4(0.4) \\
0.4(0.3) \\
0.14(0.06) \\
\\
2,116(155) \\
3,786(169)
\end{array}
$$

$$
\begin{array}{r}
703(245) \\
460(124) \\
323(185) \\
516(102) \\
620(202) \\
228(127) \\
240(140) \\
169(124) \\
238(166) \\
153(34) \\
78(16)
\end{array}
$$

$$
\begin{array}{rr}
5839 & (1679) \\
5837 & (1938) \\
5645 & (1044) \\
5186 & (3520) \\
3660 & (1515) \\
3272 & (1568) \\
1677 & (1662) \\
499 & (224) \\
317 & (158) \\
310 & (299) \\
299 & (140) \\
257 & (126) \\
248 & (225) \\
131 & (66) \\
12 & (12) \\
7 & (7) \\
7 & (7) \\
7 & (7) \\
0.4 & (0.3) \\
0.15 & (0.06)
\end{array}
$$

120,485 (11264)

183,742 (9641)

51,478 (21338) 30,313 (12505)

18,582 (10984)

17,895 (4906)

14,823 (4798)

$14,277 \quad(8671)$

14,025 (8224)

$13,383 \quad(9420)$

12,074 (8800)

9,124 (1911)

5,962 (1634)
$0.182(0.036)$

$0.402(0.095)$

$0.308(0.058)$

$0.153(0.111)$

$0.101(0.035)$

0.235 (0.109)

$0.062(0.059)$

$0.017(0.007)$

$0.028(0.014)$

$0.024(0.022)$

$0.023(0.010)$

0.021 (0.010)

$0.026(0.024)$

$0.012(0.006)$

$0.001(0.001)$

$0.001(0.001)$

$0.001(0.001)$

$0.001(0.001)$

0.001 (0.001)

$0.0002(0.0001)$

$2.802(0.208)$

$5.376(0.195)$

0.998 (0.349)

$0.589(0.159)$

$0.413(0.237)$

$0.902(0.178)$

$0.477(0.155)$

$0.374(0.208)$

$0.468(0.274)$

$0.312(0.231)$

$0.305(0.213)$

$0.400(0.088)$

$0.199(0.040)$ 
Robinia pseudoacacia

Pinus strobus

Quercus alba

Cornus florida

Castanea dentata

Sassafras albidum

Hamamelis virginiana

Rhododendron maximum

Amelanchier arborea

Acer pensylvanicum

Tsuga canadensis

Castanea pumila

Magnolia fraseri

Fagus grandifolia

Magnolia acuminata

Pyrularia pubera

$R$. calendulaceum

All Quercus

Total

204 (42)

5196 (1337)

\section{Species in 2009}

Quercus montana

Quercus rubra

Acer rubrum

Quercus coccinea

Pinus rigida

Liriodendron tulipifera

Carya spp.

Kalmia latifolia

Oxydendrum arboretum

Nyssa sylvatica

Robinia pseudoacacia

Quercus alba

$\begin{array}{rrrr}30(10) & 0.74(0.32) & 88 & (38) \\ 4(4) & 0.62(0.62) & 65 & (65) \\ 9(22) & 0.40(0.39) & 54 & (71) \\ 96(33) & 0.65(0.32) & 74 & (26) \\ 89(12) & 0.34(0.14) & 39 & (9) \\ 43(4) & 0.15(0.09) & 17 & (9) \\ 91(20) & 0.20(0.16) & 23 & (7) \\ 38(24) & 0.38(0.26) & 14 & (9) \\ 14(8) & 0.08(0.05) & 9 & (10) \\ 5(4) & 0.03(0.02) & 6 & (4) \\ 5(4) & 0.03(0.02) & 11 & (9) \\ 20(20) & 0.04(0.04) & 5 & (5) \\ 9(3) & 0.02(0.01) & 3 & (1) \\ 4(2) & 0.01(0.01) & 16(10) \\ 5(2) & 0.004(0.004) & 0.4(0.4) \\ 4(4) & 0.01(0.01) & 0.1(0.06) \\ 4(4) & 0.01(0.01) & 0.02(0.02)\end{array}$

1,778 (394)

4,163 (422)

$$
\begin{array}{r}
475(130) \\
468(248) \\
632(107) \\
405(133) \\
337(210) \\
209(158) \\
276(120) \\
542(191) \\
152(32) \\
86(13) \\
67(33) \\
67(66)
\end{array}
$$

$$
\begin{array}{rr}
4,602 & (2271) \\
3,474 & (3474) \\
2,392 & (2383) \\
2,213 & (1156) \\
1,221 & (732) \\
451 & (258) \\
447 & (358) \\
359 & (241) \\
233 & (164) \\
133 & (86) \\
123 & (108) \\
86 & (86) \\
54 & (29) \\
16 & (10) \\
8 & (8) \\
0.02 & (0.02) \\
0.02 & (0.02)
\end{array}
$$

114,840 (29105) 218,356 (29985)

$0.120(0.052)$

$0.119(0.119)$

$0.079(0.077)$

$0.149(0.074)$

$0.066(0.029)$

$0.029(0.018)$

$0.046(0.037)$

$0.011(0.007)$

$0.015(0.010)$

$0.011(0.007)$

$0.008(0.006)$

0.008 (0.008)

$0.009(0.004)$

$0.001(0.001)$

$0.001(0.001)$

$<0.001$

$<0.001$

$2.385(0.521)$

$6.151(0.551)$

$\begin{aligned} 30,050(10165) & 0.676(0.185) \\ 27,514(14470) & 0.599(0.317) \\ 25,849(6205) & 1.106(0.187) \\ 25,051 \quad(9099) & 0.518(0.171) \\ 18,836(11662) & 0.636(0.395) \\ 18,812(13746) & 0.387(0.293) \\ 17,712(8396) & 0.454(0.196) \\ 12,977(4546) & 0.418(0.147) \\ 9,444(2092) & 0.396(0.085) \\ 7,210(1698) & 0.220(0.033) \\ 3,691 \quad(1980) & 0.091(0.045) \\ 3,689(3678) & 0.099(0.097)\end{aligned}$




\begin{tabular}{|c|c|c|c|c|c|}
\hline Quercus velutina & $6(3)$ & $0.31(0.16)$ & $38(21)$ & $1,841(1059)$ & $0.049(0.027)$ \\
\hline Cornus florida & $28(6)$ & $0.42(0.18)$ & $48(21)$ & 1,457 (688) & $0.097(0.042)$ \\
\hline Rhododendron maximum & $233(131)$ & $0.96(0.59)$ & $52(31)$ & 1,303 (767) & $0.040(0.024)$ \\
\hline Amelanchier arborea & $19(7)$ & $0.54(0.11)$ & $31(13)$ & 1,264 (586) & $0.051(0.022)$ \\
\hline Sassafras albidum & $45(41)$ & $0.15(0.14)$ & $18(18)$ & $455(450)$ & $0.030(0.029)$ \\
\hline Acer pensylvanicum & $12(5)$ & $0.06(0.05)$ & $11(7)$ & 270 & $0.020(0.012)$ \\
\hline Magnolia fraseri & 19 (6) & $0.06(0.11)$ & 7 (3) & $177 \quad(84)$ & $0.024(0.011)$ \\
\hline Symplocos tinctoria & $14(6)$ & $0.05(0.03)$ & 5 (3) & 142 (99) & $0.009(0.005)$ \\
\hline Hamamelis virginiana & $40(34)$ & $0.05(0.04)$ & 6 (5) & 110 (93) & $0.013(0.011)$ \\
\hline Castanea dentata & $20(11)$ & $0.03(0.02)$ & 4 (2) & $70 \quad(40)$ & $0.006(0.003)$ \\
\hline Betula lenta & $3(2)$ & $0.02(0.01)$ & 1 (1) & $60 \quad(53)$ & $0.002(0.002)$ \\
\hline Magnolia acuminata & $5(3)$ & $0.02(0.01)$ & 2 (2) & 54 (45) & $0.007(0.005)$ \\
\hline Fagus grandifolia & $5(2)$ & $0.01(0.005)$ & 1 (1) & 21 (14) & $0.002(0.001)$ \\
\hline Fraxinus americana & $2(2)$ & $0.003(0.003)$ & $0.4 \quad(0.4)$ & $8 \quad(8)$ & $0.001(0.001)$ \\
\hline Pinus strobus & $3(2)$ & $0.003(0.002)$ & $0.02(0.01)$ & $4 \quad(3)$ & $<0.001$ \\
\hline Castanea pumila & $2(2)$ & $0.002(0.002)$ & $0.2 \quad(0.2)$ & 3 (3) & $<0.001$ \\
\hline R. calendulaceum & $17(8)$ & $0.02(0.01)$ & $0.06(0.03)$ & $1(0.04)$ & $<0.001$ \\
\hline All Quercus & 167 (29) & $10.25(1.74)$ & $1,455(235)$ & 88,145 (15363) & $1.941(0.304)$ \\
\hline Total & 4635 (1289) & 34.37 (3.06) & $3,944(312)$ & 208,079 (13518) & $5.951(0.394)$ \\
\hline
\end{tabular}

In 1934, only percent cover of evergreen shrubs (Kalmia latifolia and Rhododendron maximum) was estimated for each plot. For all other years, diameter of evergreen shrubs was measured. 


\section{Table S6}

Watershed 6: mean density, basal area, aboveground biomass, and leaf area index (LAI) of species over time: before (1934, 1958), during (1961-1965), and after grass-to-forest-conversion (1968, 1982, 1995, 2012). Standard errors are in parentheses. Proportional contributions of dominant species to the watershed totals are in brackets.

\begin{tabular}{|c|c|c|c|c|c|}
\hline \multirow[b]{2}{*}{ Species in 1934} & \multirow{2}{*}{$\begin{array}{c}\text { Density } \\
\left(\text { stems ha }^{-1}\right)\end{array}$} & \multirow{2}{*}{$\begin{array}{c}\text { Basal area } \\
\left(\mathrm{m}^{2} \mathrm{ha}^{-1}\right)\end{array}$} & \multicolumn{2}{|c|}{ Aboveground biomass $\left(\mathrm{kg} \mathrm{ha}^{-1}\right)$} & \multirow{2}{*}{$\begin{array}{c}\text { LAI } \\
\left(\mathrm{m}^{2} \mathrm{~m}^{-2}\right)\end{array}$} \\
\hline & & & Leaf & Total & \\
\hline Castanea dentata & $600(162)$ & $11.99(3.57)$ & $1,443(430)$ & $102,386(31647)$ & $2.395(0.714)$ \\
\hline Quercus montana & 320 (118) & $3.77(1.20)$ & $568(209)$ & $40,294(18605)$ & $0.806(0.296)$ \\
\hline Quercus coccinea & $322(76)$ & $3.35(1.12)$ & $908(214)$ & $23,031(8900)$ & $1.162(0.274)$ \\
\hline Carya spp. & 268 (53) & $1.95(0.61)$ & $238(85)$ & $14,615(5688)$ & $0.391(0.140)$ \\
\hline Pinus rigida & 25 (17) & $1.98(1.23)$ & $179(112)$ & $10,040(6241)$ & $0.339(0.211)$ \\
\hline Acer rubrum & $498(184)$ & $1.33(0.36)$ & 279 (79) & $4,914(1378)$ & $0.488(0.138)$ \\
\hline Nyssa sylvatica & $230(80)$ & $1.04(0.48)$ & 92 (27) & $4,659(3411)$ & $0.234(0.070)$ \\
\hline Oxydendrum arboreum & 180 & $0.77(0.11)$ & 81 (12) & $2,167 \quad(296)$ & $0.212(0.031)$ \\
\hline Quercus velutina & 50 & $0.35(0.11)$ & 36 (14) & 1,466 (633) & $0.047(0.018)$ \\
\hline Robinia pseudoacacia & 40 (20) & $0.28(0.14)$ & 32 (17) & 1,047 (593) & $0.044(0.023)$ \\
\hline Cornus florida & 135 (56) & $0.35(0.18)$ & 39 (20) & $804(470)$ & $0.078(0.041)$ \\
\hline Quercus alba & 75 (25) & $0.25(0.12)$ & 54 (24) & 698 (420) & $0.079(0.035)$ \\
\hline Amelanchier arborea & 52 (14) & $0.11(0.03)$ & 12 & 231 & $0.020(0.005)$ \\
\hline Quercus rubra & 20 & $0.06(0.03)$ & $4 \quad(2)$ & 136 (70) & $0.006(0.003)$ \\
\hline Quercus falcata & $8 \quad(5)$ & $0.03(0.02)$ & 19 (11) & $97 \quad$ (68) & $0.024(0.014)$ \\
\hline Castanea pumila & $15 \quad$ (9) & $0.03(0.02)$ & $4 \quad(2)$ & $66 \quad(40)$ & $0.006(0.004)$ \\
\hline Sassafras albidum & 10 & $0.02(0.01)$ & (1) & $44 \quad$ (21) & $0.004(0.002)$ \\
\hline Diospyros virginiana & $5 \quad(5)$ & $0.03(0.01)$ & (1) & $22 \quad(22)$ & $0.002(0.002)$ \\
\hline All Quercus & 787 (104) & $7.78(2.12)$ & $1,569(404)$ & 65,626 (24887) & 2.122 (0.559) \\
\hline Total & 4302 (213) & $28.39(2.61)$ & $4,134(377)$ & 209,855 (20713) & $6.447(0.603)$ \\
\hline \multicolumn{6}{|l|}{ Species in 1958} \\
\hline Quercus montana & 165 & $3.14(0.65)$ & 470 (103) & 32,321 (8492) & $0.667(0.146)$ \\
\hline Quercus coccinea & 88 (16) & $2.21(0.47)$ & 418 (71) & 15,557 (1079) & $0.536(0.091)$ \\
\hline Acer rubra & 284 (42) & $1.38(0.27)$ & 244 (41) & $8,593(2526)$ & $0.428(0.072)$ \\
\hline
\end{tabular}


Carya spp.

Pinus rigida

Quercus velutina

Rhododendron maximum

Quercus alba

Kalmia latifolia

Nyssa sylvatica

Oxydendrum arboreum

Quercus rubra

Cornus florida

Robinia pseudoacacia

Liriodendron tulipifera

Betula lenta

Hamamelis virginiana

Fagus grandifolia

Sassafras albidum

Castanea dentata

Amelanchier arborea

Tsuga canadensis

Symplocos tinctoria

Diospyros virginiana

Magnolia acuminata

Fraxinus americana

Salix nigra

Castanea pumila

Juglans cinerea

Acer pensylvanicum

$R$. calendulaceum

Cleathra acuminata

Pyrularia pubera

Calycanthus florida

Vaccinium stamineum

Cornus alternifolia

All Quercus

Total

\begin{tabular}{|c|c|c|}
\hline $186 \quad(26)$ & $1.27(0.26)$ & 145 (34) \\
\hline (6) & $1.13(0.52)$ & $98 \quad(46)$ \\
\hline (9) & $0.89(0.27)$ & 112 (37) \\
\hline $1893(270)$ & $1.00(0.15)$ & 188 (27) \\
\hline $80 \quad(24)$ & $0.80(0.28)$ & 126 (39) \\
\hline 1827 (338) & $0.94(0.17)$ & 181 (33) \\
\hline $98 \quad(18)$ & $0.68(0.17)$ & 51 (10) \\
\hline 105 (14) & $0.63(0.11)$ & (9) \\
\hline $20 \quad(7)$ & $0.28(0.14)$ & 36 (18) \\
\hline 240 & 0.50 (0.09) & 56 (11) \\
\hline 32 (10) & $0.17(0.04)$ & 19 \\
\hline 138 & $0.18(0.05)$ & 16 \\
\hline 215 (69) & $0.20(0.05)$ & (2) \\
\hline 153 (65) & $0.13(0.05)$ & 15 \\
\hline (6) & $0.05(0.03)$ & (4) \\
\hline 55 (27) & $0.06(0.02)$ & (3) \\
\hline $31 \quad$ (9) & $0.06(0.02)$ & (2) \\
\hline $22 \quad(5)$ & $0.04(0.01)$ & (1) \\
\hline $7 \quad$ (4) & $0.02(0.01)$ & 11 \\
\hline 10 & $0.02(0.01)$ & (1) \\
\hline 2 (1) & $<0.01(0.01)$ & $1(0.6)$ \\
\hline $7 \quad(2)$ & $0.01(0.004)$ & $1(0.5)$ \\
\hline 12 (7) & $0.01(0.01)$ & 1 (1) \\
\hline 10 (10) & $0.01(0.01)$ & 0.59 (0.59) \\
\hline $1(0.8)$ & $<0.01(0.01)$ & $0.33(0.18)$ \\
\hline 2 (1) & $<0.01(0.01)$ & $0.16(0.14)$ \\
\hline $1 \quad$ (1) & $0.01(0.01)$ & $0.20(0.20)$ \\
\hline $97 \quad(24)$ & $0.05(0.01)$ & $0.14(0.04)$ \\
\hline $62 \quad$ (33) & $0.03(0.02)$ & $0.09(0.05)$ \\
\hline 14 (7) & $0.03(0.01)$ & $0.07(0.04)$ \\
\hline $6 \quad$ (4) & $<0.01(0.01)$ & $0.01(0.01)$ \\
\hline $6 \quad$ (4) & $<0.01(0.01)$ & $0.01(0.01)$ \\
\hline $5 \quad(2)$ & $<0.01(0.003)$ & $0.65(0.32)$ \\
\hline $393(34)$ & $7.33(1.05)$ & $1,162(160)$ \\
\hline 5942 (292) & 15.96 (1.38) & $2,283(206)$ \\
\hline
\end{tabular}

$\begin{array}{rrr}8,182(2194) & 0.238(0.056) \\ 5,621(2617) & 0.189(0.088) \\ 5,394(1856) & 0.143(0.047) \\ 4,128(592) & 0.145(0.021) \\ 4,112(1757) & 0.185(0.057) \\ 3,692(732) & 0.139(0.026) \\ 3,592(1292) & 0.131(0.025) \\ 2,203(440) & 0.148(0.024) \\ 1,802(912) & 0.046(0.023) \\ 1,317(305) & 0.113(0.021) \\ 697(210) & 0.026(0.007) \\ 454 & (136) & 0.029(0.008) \\ 435(110) & 0.015(0.004) \\ 236 & (89) & 0.030(0.011) \\ 222 & (165) & 0.010(0.006) \\ 206 & (131) & 0.012(0.005) \\ 119 & (39) & 0.009(0.003) \\ 111 & (32) & 0.011(0.003) \\ 102 & (59) & 0.007(0.004) \\ 36 & (17) & 0.003(0.001) \\ 27 & (18) & 0.002(0.001) \\ 24 & (9) & 0.004(0.002) \\ 18 & (11) & 0.002(0.001) \\ 7 & (7) & 0.001(0.001) \\ 6 & (3) & 0.001(0.001) \\ 5 & (4) & 0.0003(0.0002) \\ 3 & (3) & 0.001(0.001) \\ 0.16(0.04) & 0.0002(0.0001) \\ 0.10(0.05) & 0.0001(0.0001) \\ 0.08(0.04) & 0.0001(0.0001) \\ 0.01(0.01) & 0.0001(0.0001) \\ 0.01(0.01) & 0.0001(0.0001) \\ 0.01(0.01) & 0.001(0.001) \\ & & \\ 9,187(11048) & 1.577(0.220) \\ 9,508(12110) & 3.273(0.294)\end{array}$


1961 - Grass
1962 - Grass
1963 - Grass
1965 - Grass

1968 - Grasses \& forbs

\section{Species in 1982}

Robinia pseudoacacia

Liriodendron tulipifera

Betula lenta

Acer rubrum

Juglans cinerea

Sassafras albidum

Carya spp.

Pinus rigida

Prunus serotina

Diospyros virginiana

Amelanchier arborea

Oxydendrum arboreum

Quercus montana

Cornus florida

Quercus alba

Symplocos tinctoria

Rhododendron maximum

Quercus rubra

Pinus strobus

Quercus velutina

Salix nigra

Platanus occidentalis

Fraxinus americana

Hamamelis virginiana

Kalmia latifolia

Tilia americana

Vitis spp.
$\mathrm{SLA}=253 ; \mathrm{LWR}=0.51$ from

Poorter and Remkes (1990) for Festuca ovina

$$
\begin{array}{rr}
7,300 & (276) \\
6,080 & (201) \\
5,670 & (932) \\
6,490 & (1067)
\end{array}
$$

3,918 (390)

$\begin{array}{rr}29,501 & (3466) \\ 9,745 & (1853) \\ 2,284 & (1032) \\ 732 & (178) \\ 707 & (707) \\ 372 & (171) \\ 367 & (128) \\ 300 & (212) \\ 273 & (148) \\ 254 & (107) \\ 217 & (154) \\ 200 & (66) \\ 164 & (106) \\ 98 & (38) \\ 85 & (85) \\ 62 & (34) \\ 52 & (38) \\ 37 & (24) \\ 35 & (34) \\ 29 & (15) \\ 21 & (21) \\ 12 & (10) \\ 5 & (5) \\ 5 & (5) \\ 3 & (3) \\ 2 & (2) \\ 0.3 & (0.1)\end{array}$

9.419

7.843

7.317

8.374

$6.462(0.644)$

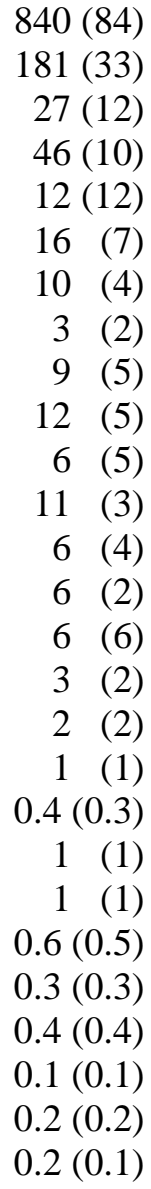

$1.142(0.115)$

$0.336(0.062)$

$0.072(0.032)$

$0.080(0.017)$

$0.019(0.019)$

$0.027(0.012)$

$0.017(0.006)$

$0.009(0.006)$

$0.015(0.007)$

$0.015(0.006)$

$0.011(0.008)$

0.030 (0.009)

0.009 (0.006)

$0.011(0.004)$

0.009 (0.009)

$0.005(0.002)$

$0.002(0.001)$

$0.002(0.001)$

$0.001(0.001)$

$0.001(0.001)$

$0.002(0.002)$

$0.001(0.001)$

$0.001(0.001)$

$0.001(0.001)$

0.0001 (0.0001)

$0.0003(0.0003)$

0.0004 (0.0001) 
Rhus copallina

Sambucus canadensis

Malus angustifolia

All Quercus

Total

$\begin{array}{rrr}22(22) & 0.02(0.02) \\ 4(4) & 0.004(0.004) \\ 1 & (1) & 0.005(0.005)\end{array}$

34 (9)

1712 (121)

$0.12(0.05)$

$11.21(0.83)$

Species in 1995

Liriodendron tulipifera

Robinia pseuodacacia

Betula lenta

Acer rubrum

Juglans cinerea

Prunus serotina

Carya spp.

Sassafras albidum

Quercus montana

Amelanchier arborea

Cornus florida

Oxydendrum arboretum

Diospyros virginiana

Rhododendron maximum

Quercus alba

Pinus strobus

Kalmia latifolia

Fraxinus americana

Symplocos tinctoria

Platanus occidentalis

Quercus rubra

Pinus rigida

Quercus rubra

Hamamelis virginiana

Halesia carolina

Acer pensylvanicum

Vitis spp.

Clethra acuminata

$\begin{array}{rr}166(30) & 7.86(1.07) \\ 204(24) & 7.33(0.88) \\ 81(39) & 1.26(0.55) \\ 194(27) & 1.29(0.25) \\ 1(1) & 0.29(0.29) \\ 13(5) & 0.44(0.22) \\ 31(9) & 0.31(0.10) \\ 26(13) & 0.28(0.16) \\ 6(4) & 0.18(0.13) \\ 6(4) & 0.15(0.10) \\ 44(14) & 0.24(0.08) \\ 42(12) & 0.24(0.08) \\ 12(7) & 0.12(0.06) \\ 147(60) & 0.17(0.06) \\ 7(6) & 0.10(0.09) \\ 4(2) & 0.11(0.07) \\ 94(60) & 0.10(0.07) \\ 7(4) & 0.06(0.04) \\ 18(6) & 0.04(0.02) \\ 3(2) & 0.02(0.02) \\ 4(2) & 0.02(0.01) \\ 1(1) & 0.01(0.01) \\ 3(2) & 0.01(0.01) \\ 7(6) & 0.01(0.01) \\ 1(1) & 0.003(0.003) \\ 1(1) & 0.001(0.001) \\ 144(26) & 0.17(0.03) \\ 12(8) & 0.01(0.01)\end{array}$

$$
\begin{array}{r}
0.1(0.1) \\
0.01(0.01) \\
0.01(0.01)
\end{array}
$$

1204 (88)

$651 \quad(89)$

$875(105)$

86 (36)

235 (43)

47 (47)

$53(27)$

31 (10)

33 (18)

25 (18)

17 (12)

28 (9)

24 (8)

14 (8)

$20 \quad(8)$

$18(16)$

5 (3)

12 (8)

8 (5)

5 (3)

3 (2)

2 (1)

$0.4(0.4)$

$0.9(0.8)$

1 (1)

$0.3 \quad(0.3)$

$0.2 \quad(0.2)$

$0.4(0.07)$

$0.04(0.02)$

$$
\begin{array}{rr}
0.1 & (0.1) \\
0.01 & (0.01) \\
0.01 & (0.01)
\end{array}
$$

314 (137)

45,563 (3761)

52,132 (7157)

45,123 (5872)

7,820 (3267)

6,498 (1415)

3,565 (3565)

2,832 (1574)

1,354 (473)

1,179 (683)

1,136 (847)

796 (554)

740 (246)

$731 \quad$ (266)

519 (316)

468 (184)

$370 \quad(354)$

358 (224)

268 (174)

255 (182)

$147 \quad(109)$

95 (85)

63 (47)

39 (39)

29 (26)

22 (21)

6 (6)

2 (2)

$0.5 \quad(0.1)$

$0.4 \quad(0.3)$
0.0001 (0.0001)

$<0.0001$

$<0.0001$

$0.021(0.011)$

$1.823(0.128)$

$1.205(0.164)$

$1.190(0.142)$

$0.234(0.098)$

$0.412(0.075)$

$0.077(0.077)$

$0.088(0.044)$

$0.050(0.017)$

$0.056(0.031)$

$0.035(0.025)$

$0.029(0.020)$

$0.056(0.018)$

$0.063(0.021)$

$0.023(0.012)$

$0.015(0.006)$

$0.026(0.024)$

$0.011(0.007)$

0.009 (0.006)

$0.013(0.009)$

$0.008(0.005)$

$0.005(0.004)$

$0.002(0.001)$

$0.001(0.001)$

0.001 (0.001)

0.002 (0.002)

$0.001(0.001)$

$0.0004(0.0004)$

$0.001(0.0001)$

0.0001 (0.0001) 
Rhus copallina

Malus angustifolia

Pyrularia pubera

Sambucus canadensis

All Quercus

Total

\section{Species in 2012}

Liriodendron tulipifera

Robinia pseudoacacia

Acer rubrum

Betula lenta

Juglans cinerea

Prunus serotina

Quercus montana

Oxydendrum arboretum

Sassafras albidum

Carya spp.

Fraxinus Americana

Diospyros virginiana

Rhododendron maximum

Pinus strobus

Cornus florida

Cornus alternifolia

Quercus alba

Hamamelis virginiana

Kalmia latifolia

Platanus occidentalis

Quercus rubra

Acer pensylvanicum

Symplocos tinctoria

Malus angustifolia

Ligustrum sinense

Castanea dentata

Clethra acuminata

$\begin{array}{rrrr}40(24) & 0.12(0.07) & 0.3(0.2) \\ 1 \quad(1) & 0.02(0.02) & 0.04(0.04) \\ 10(7) & 0.01(0.01) & 0.02(0.01) \\ 1(1) & <0.001 & <0.01\end{array}$

1 (1)

21 (7)

$1338(142)$

$0.32(0.15)$

21.00 (1.45)

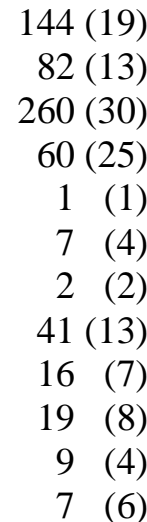

7 (6)

150 (51)

3 (2)

12 (5)

3 (3)

3 (2)

25 (17)

54 (25)

1 (1)

6 (3)

1 (1)

7 (4)

1 (1)

7 (7)

4 (4)

24 (24)

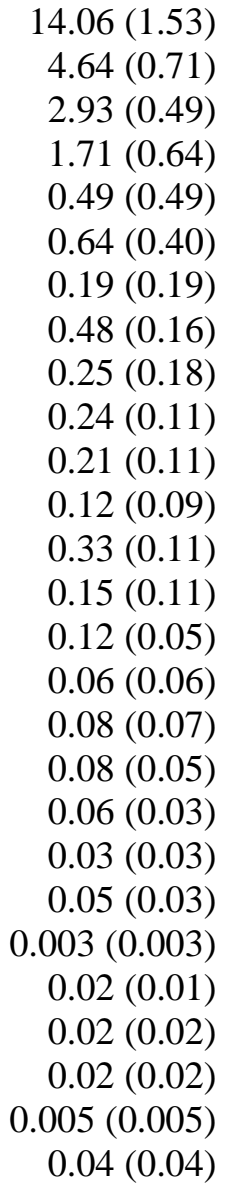

$$
\begin{array}{r}
0.3(0.1) \\
0.04(0.04) \\
0.02(0.02) \\
<0.01
\end{array}
$$

$46 \quad(23)$

2196 (142)

$1,598(900)$

126,998 (9618)

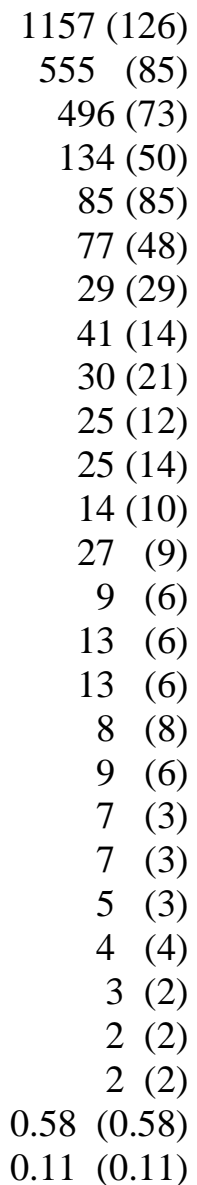

$\begin{array}{rr}115,093 & (12904) \\ 32,210 & (5287) \\ 18,314 & (3584) \\ 12,329 & (4562) \\ 7,221 & (7221) \\ 5,225 & (3465) \\ 1,838 & (1838) \\ 1,772 & (616) \\ 1,248 & (990) \\ 1,194 & (602) \\ 1,057 & (607) \\ 650 & (535) \\ 650 & (218) \\ 599 & (430) \\ 429 & (204) \\ 429 & (204) \\ 387 & (387) \\ 203 & (141) \\ 175 & (80) \\ 175 & (80) \\ 169 & (101) \\ 141 & (141) \\ 73 & (46) \\ 62 & (62) \\ 43 & (43) \\ 9 & (9) \\ 9 & (9) \\ & \end{array}$

0.0005 (0.0003)

0.0001 (0.0001)

0.0001 (0.0001) $<0.0001$

$0.065(0.034)$

$3.616(0.241)$

$1.840(0.200)$

0.689 (0.106)

$0.714(0.113)$

$0.166(0.061)$

$0.140(0.140)$

$0.100(0.063)$

$0.036(0.036)$

$0.081(0.027)$

$0.038(0.028)$

$0.036(0.017)$

$0.032(0.018)$

$0.018(0.013)$

$0.008(0.003)$

$0.019(0.014)$

$0.027(0.012)$

$0.027(0.012)$

$0.016(0.016)$

$0.012(0.008)$

$0.002(0.001)$

$0.002(0.001)$

$0.006(0.003)$

$0.006(0.006)$

$0.005(0.003)$

$0.003(0.003)$

$0.003(0.003)$

$0.001(0.001)$

0.0002 (0.0002) 


\begin{tabular}{|c|c|c|c|c|c|}
\hline Juniperus virginiana & $3(2)$ & $0.005(0.004)$ & $0.05(0.04)$ & (6) & $0.0002(0.0001)$ \\
\hline Vitis spp. & 201 (29) & $0.31(0.12)$ & $0.82(0.12)$ & $0.9(0.1)$ & $0.001(0.0002)$ \\
\hline Aristolocchia macrophylla & $3(3)$ & $0.002(0.002)$ & $0.01(0.01)$ & $0.01(0.01)$ & $<0.0001$ \\
\hline R. calendulaceum & 6 (5) & $0.006(0.005)$ & $0.02(0.01)$ & $0.02(0.01)$ & $<0.0001$ \\
\hline Parthenocissus quinquefolia & 4 (2) & $0.006(0.004)$ & $0.02(0.01)$ & $0.02(0.01)$ & $<0.0001$ \\
\hline Pyrularia pubera & 3 (3) & $0.003(0.003)$ & $0.01(0.01)$ & $0.01(0.01)$ & $<0.0001$ \\
\hline All Quercus & 10 & $0.31(0.20)$ & $45(30)$ & 2,367 (1854) & $0.064(0.043)$ \\
\hline Total & $1174(90)$ & $27.36(1.81)$ & 2770 (185) & $201,470(14665)$ & $4.848(0.330)$ \\
\hline
\end{tabular}

In 1934, only percent cover of evergreen shrubs (Kalmia latifolia and Rhododendron maximum) was estimated for each plot. For all other years, diameter of evergreen shrubs was measured. 


\section{Figure S1}

Mean ( \pm SE bars) aboveground biomass for the dominant species in the (a) treated WS6, (b) reference WS14, and (c) reference WS18 over time. Dominant species were Castanea dentata, Quercus (montana, coccinea, rubra, velutina, alba), Carya spp., Acer rubrum, Liriodendron tulipifera, Robinia pseudoacacia, and Betula lenta.

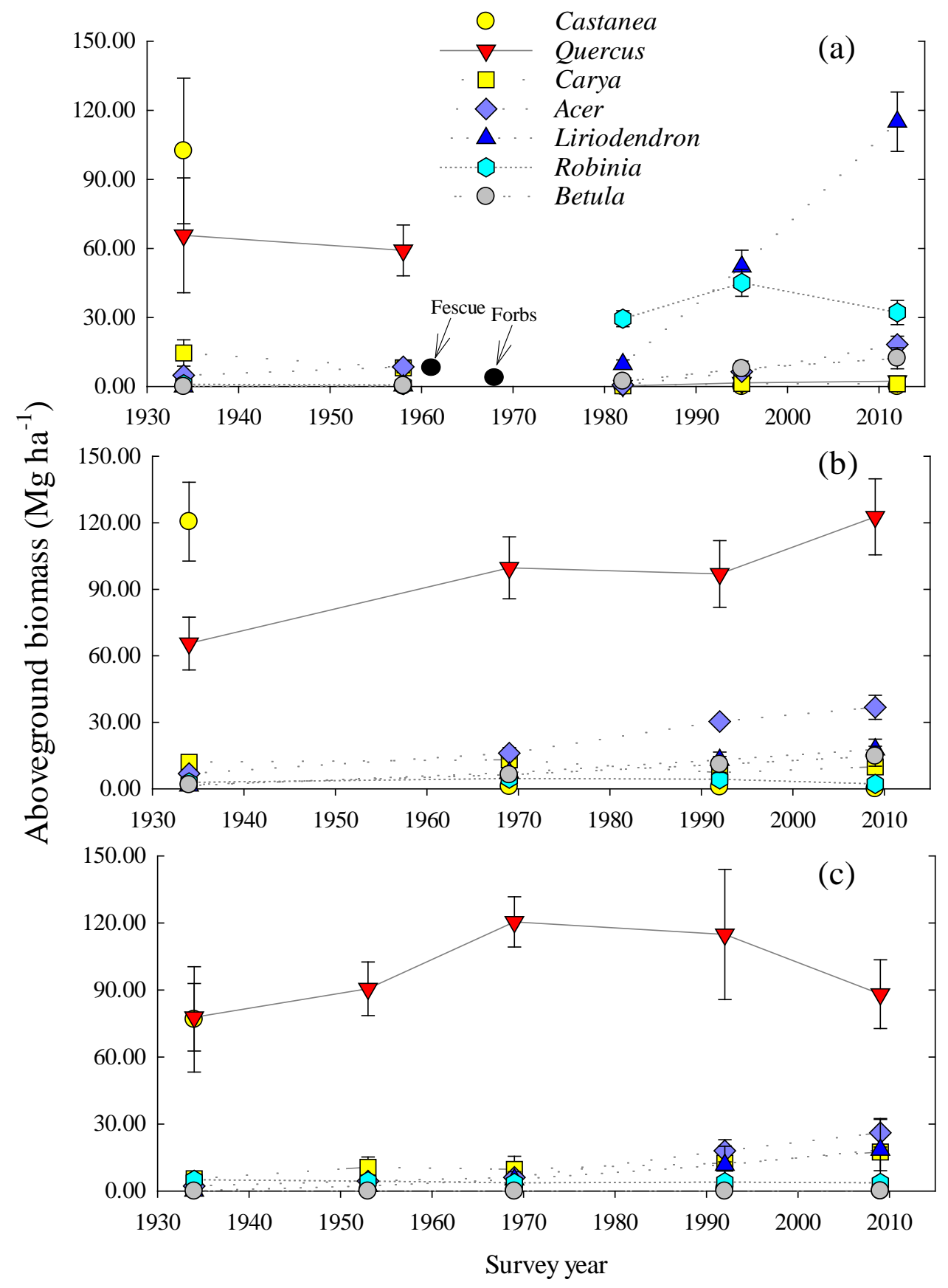




\section{Figure S2}

Change in evapotranspiration $\left(\boldsymbol{D}_{\mathrm{ET}}\right.$, calculated as $P-\hat{Q}_{T}$; where, $\boldsymbol{D}_{\boldsymbol{E} \boldsymbol{T}}=\left[P-Q_{T}\right]-$ $\left.\left(\left[P-\hat{Q}_{T}\right] ; M 1, M 2=0\right)\right)$ for the treated WS6 over time (bars). Solid lines are the standard errors of the mean prediction. We used the paired-watershed approach with WS18 as the reference. The year of harvest, conversion to Kentucky-31 fescue grass (Festuca octiflora) cover, fertilize, herbicide, and abandonment to allow forest regeneration are denoted by dashed lines.

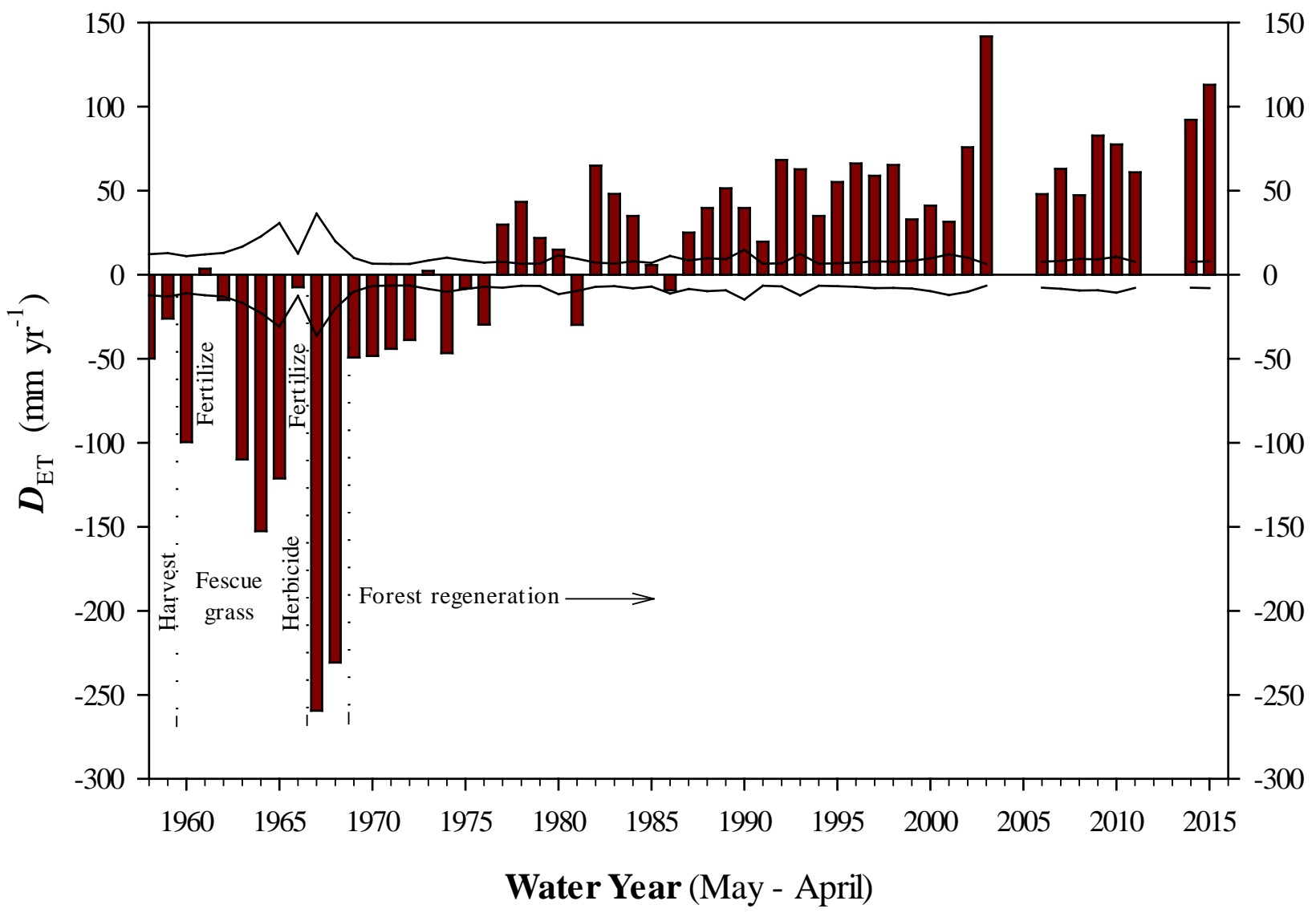

